\title{
Estrogen Shapes Dopamine-Dependent Cognitive Processes: Implications for Women's Health
}

\author{
Emily Jacobs and Mark D’Esposito \\ Henry H. Wheeler Jr. Brain Imaging Center, Helen Wills Neuroscience Institute, University of California, Berkeley, Berkeley, California 94720
}

\begin{abstract}
The prefrontal cortex (PFC) is exquisitely sensitive to its neurochemical environment. Minor fluctuations in cortical dopamine (DA) can profoundly alter working memory, a PFC-dependent cognitive function that supports an array of essential human behaviors. Dopamine's action in the PFC follows an inverted U-shaped curve, where an optimal DA level results in maximal function and insufficient or excessive DA impairs PFC function. In animals, $17 \beta$-estradiol (the major estrogen in most mammals, referred to henceforth as estradiol) has been shown to enhance DA activity, yet no human study has adequately addressed whether estradiol's impact on cognition occurs by way of modulating specific neurochemical systems. Here we examined the effects of endogenous fluctuations in estradiol on working memory in healthy young women as a function of baseline PFC DA [indexed by catechol- $O$-methyltransferase (COMT) Val ${ }^{158} \mathrm{Met}$ genotype and, at a finer scale, COMT enzyme activity]. The results demonstrate that estradiol status impacts working memory function and, crucially, the direction of the effect depends on indices of baseline DA. Moreover, consistent with a DA cortical efficiency hypothesis, functional MRI revealed that inferred optimal DA was associated with reduced PFC activity sustained across task blocks and selectively enhanced PFC activity on trials with the greatest demand for cognitive control. The magnitude of PFC activity during high control trials was predictive of an individual's performance. These findings show that although estrogen, considered in isolation, may have unpredictable effects on cognitive performance, its influence is clarified when considered within a larger neuromodulatory framework. Given the clinical prevalence of dopaminergic drugs, understanding the relationship between estrogen and DA is essential for advancing women's health.
\end{abstract}

\section{Introduction}

Working memory (WM) is a fundamental cognitive ability that supports an array of complex abilities, from problem solving to fluid intelligence (Barrett et al., 2004). Dopamine (DA) signaling in prefrontal cortex (PFC) is critical for WM function (Brozoski et al., 1979; Sawaguchi and Goldman-Rakic, 1991; Williams and Goldman-Rakic, 1995). Dopamine's relationship to performance on frontally mediated cognitive tasks is not linear; DA function follows an inverted U-shaped curve, where optimal DA results in maximal function and insufficient or excessive levels lead to PFC dysfunction (Cai and Arnsten, 1997; Zahrt et al., 1997; Granon et al., 2000; Egan et al., 2001; Gibbs and D'Esposito, 2005a; Vijayraghavan et al., 2007; Williams-Gray et al., 2007). Thus, taking basal DA levels into account is essential for predicting how DA augmentation (e.g., via a drug) will affect cognitive performance (Egan et al., 2001).

Individual differences in baseline PFC DA stem, in part, from genetic alterations that impact the DA system. The catechol-O-

\footnotetext{
Received Dec. 8, 2010; revised Feb. 9, 2011; accepted Feb. 11, 2011.

Author contributions: E.J. and M.D. designed research; E.J. and M.D. performed research; E.J. analyzed data; E.J. and M.D. wrote the paper.

This work was supported by the National Institutes of Health (M.D.), a National Science Foundation Graduate Research Fellowship (E.J.), and the Berkeley Consortium on Population Health and Human Development- Robert Wood Johnson Foundation Health and Society Scholars Program (E.J.). We thank Daniel Weinberger, Bhaskar Kolachana, and Jingshan Chen for advice on and analysis of COMT enzyme data and Lance Kriegsfeld for help with the estradiol ELISA.

Correspondence should be addressed to Emily Jacobs, Center for Brain Science, Harvard University, Northwest Science East Wing, Room 210-05, 52 0xford Street, Cambridge, MA 02138. E-mail: emily.jacobs@gmail.com. DOI:10.1523/JNEUROSCI.6394-10.2011

Copyright $\odot 2011$ the authors $\quad 0270-6474 / 11 / 315286-08 \$ 15.00 / 0$
}

methyltransferase (COMT) gene codes for an enzyme that metabolizes released DA. COMT enzyme is crucial for regulating frontal DA transmission, accounting for $>60 \%$ of total DA turnover in PFC [compared with $15 \%$ in the striatum (Männistö and Kaakkola, 1999)]. A recent COMT gene polymorphism ( $\mathrm{Val}^{158} \mathrm{Met}$ ) alters the efficiency of the eponymous enzyme; compared with the ancestral $\mathrm{val} / \mathrm{val}$ allelic variant, met/met individuals have decreased COMT activity, enhanced PFC-dependent cognitive function, and greater cortical efficiency (Egan et al., 2001; Tunbridge et al., 2006).

In animals, strong evidence indicates that estradiol enhances DA activity on rapid and protracted timescales (Thompson and Moss, 1994; Becker, 2000). Estradiol enhances DA synthesis, release, and turnover and modifies basal firing rates of DA neurons via membrane estrogen receptors (Xiao and Becker, 1994; Pasqualini et al., 1995; Becker, 1990, 2000). Some evidence links estrogen and WM function; for example, improvements in WM have been observed in postmenopausal women on estrogen replacement compared with nonusers, but the data are inconsistent (Duff and Hampson, 2000; Sherwin, 2005). Some evidence suggests that WM span fluctuates throughout the estrogen cycle (Rosenberg and Park, 2002), but here too the data are inconsistent (Gasbarri et al., 2008). The complicated relationship between estrogen and WM may stem from unaccounted variability in baseline DA across individuals. Accordingly, a study in rats showed that the administration of a DA drug had different effects on WM performance depending on the rats' estrous stage at the time of testing (Shansky et al., 2004).

Evidence for an estradiol-DA link exists in animals, yet no human study has examined whether DA mediates estradiol's ef- 
fects on PFC function. Given that estradiol levels may be higher in the PFC than any other cortical area (Bixo et al., 1995), it is likely that estradiol's effects on WM function are mediated, in part, through modulation of PFC DA activity. Thus, we predicted that performance on tasks that are sensitive to PFC DA signaling would vary throughout the menstrual cycle. Importantly, we predicted that estradiol's behavioral effects would not be consistent unless individual variation in baseline DA was accounted for. Here we examine estradiol's effects on WM behavior and neural activity as a function of COMT genotype and enzyme activity.

\section{Materials and Methods}

Subjects. Seventy-nine healthy female participants (mean age, $21.7 \pm 2.4$ years) were recruited via advertisements on the UC Berkeley campus and prescreened for COMT Val ${ }^{158}$ Met polymorphism. Exclusionary criteria included any history of neurological or psychiatric disorders, an episode of loss of consciousness, use of psychotropic drugs, a history of substance abuse, MRI contraindications, abnormal or infrequent menstrual cycle, and/or use of a hormonal birth control. Blood samples were collected by a licensed phlebotomist and sent for DNA extraction and genotyping. The menstrual cycles of homozygous participants were tracked for $\geq 4$ months (across at least four menstruation periods) to select subjects with the most regular cycles, who were then invited for inclusion in the central study. Twenty-four women were enrolled: $13 \mathrm{val} / \mathrm{val}$, eight $\mathrm{met} / \mathrm{met}$, and three $\mathrm{val} / \mathrm{met}$ (the three heterozygotes were enrolled before their genotype was known; their data are included in analyses assessing the effects of estradiol regardless of genotype). Subjects underwent both behavioral and fMRI testing on two occasions (two val/val and two val/met subjects did not return for the second session), resulting in the total acquisition of 44 datasets. Of the 24 subjects, 21 had no history of smoking and three reported having previously smoked ( $>4$ months before study enrollment), albeit infrequently (less than four cigarettes a week). The study was approved by the UC Berkeley Committee for the Protection of $\mathrm{Hu}-$ man Subjects. All volunteers gave written informed consent and were paid for their participation. Ethnicity was matched between genotypic groups to minimize population stratification effects. Of met/met participants, 63\% self-identified as Asian American and 37\% identified as Caucasian American. The numbers are nearly identical for $\mathrm{val} / \mathrm{val}$ participants, with $62 \%$ Asian American and 38\% Caucasian American. (The data reflect the UC Berkeley student population from which participants were recruited.) Indices of parental socioeconomic status were not obtained; educational status did not differ between participants.

Experimental design. Following inclusion in the main study, subjects were seen on three occasions. On the first visit, exclusionary criteria were reviewed, consent for the main study was obtained, and the participants completed an introductory neuropsychological exam. The second and third visits were time-locked to each subject's menstrual cycle. One test session occurred on or near day 1 or 2 of the subject's cycle (during menses, when estradiol levels are low) and the other session occurred on day 11 or 12 (late follicular phase when estradiol levels peak); the order was counter-balanced across subjects. This timing assumes a $28 \mathrm{~d}$ cycle. The period between the start of menses and ovulation (follicular phase) can vary in duration, contributing to a longer or shorter overall cycle duration. The period from ovulation to the start of the next cycle (luteal phase) is less variable, with an average duration of $14 \mathrm{~d}$. Subjects' menstrual cycles were tracked (including start and duration of menses) for $\geq 4$ months before testing through 1 month posttesting to confirm cycles were predictable and regular. Blood samples were acquired on both test days to measure estradiol and COMT enzyme levels. Samples were collected following, at minimum, $4 \mathrm{~h}$ of no food or drink consumption (excluding water).

Neuropsychological measures. The initial neuropsychological exam included the following: (1) the North American Adult Reading Test (Nelson, 1982), (2) the Beck Depression Inventory (Beck et al., 1961), (3) a paper-and-pen version of the Stroop task (Stroop, 1935), (4) the controlled oral-word association test (average number of words generated in $60 \mathrm{~s}$ for the letters F, A, and S and categories animals and fruits) (Benton, 1968), (5) the Montreal Cognitive Assessment test (to assess mild cogni-
A

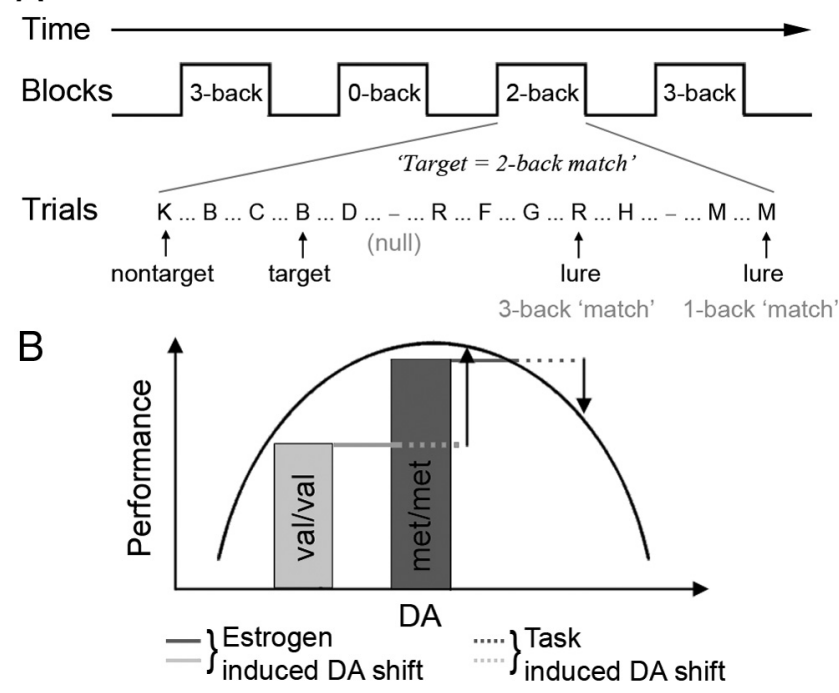

Figure 1. A, Task protocol: hybrid block/event-related design. Subjects performed blocks of WM trials with loads of 0,2 , and 3 . Within load blocks, trial types included targets (an $n$-back match), lures (a recently seen but nontarget stimulus; e.g., in the 2-back condition, a 1- or 3-back match is a lure), and nontargets (all other nonlure, nontarget trials). Stimuli (uppercase consonants) were presented for $1 \mathrm{~s}$ followed by a $1 \mathrm{~s}$ delay. Subjects responded to every trial, indicating whether the currently presented letter did or did not match the letter seen $n$-previously. The 0-back condition consisted of target-detection (the letter X). Null events provide temporal jitter for event-related analyses. $\boldsymbol{B}$, Theoretical inverted-U model of cortical DA function. Both insufficient and excessive DA-receptor stimulation lead to poor performance on DA-dependent tasks. The val/val allele of the COMT Val ${ }^{158} \mathrm{Met}$ polymorphism is associated with low basal PFC DA (light gray bar), relative to met/met carriers (dark gray bar). Estradiol (solid line) and WM load (dotted line) potentiate DA, which is expected to have beneficial effects for val homozygotes and unfavorable effects for met homozygotes.

tive impairment) (Nasreddine et al., 2005), and (6) the Barratt Impulsiveness Scale (Patton et al., 1995). Listening span (Salthouse and Babcock, 1991) and automated reading span (Daneman and Carpenter, 1980; Unsworth et al., 2005) were collected in a subsample of 19 subjects on a separate day. Genotypic groups did not differ significantly with respect to age, education, or any background neuropsychological measure.

Test day measures. During the low and high estradiol test sessions, subjects completed an $\mathrm{N}$-back verbal working memory task during fMRI scanning. To assess mood, subjects completed a positive and negative affect scale (Watson et al., 1988) and visual analog scales for the items anxious, happy, sad, nauseous, drowsy, jittery, fatigued, and dizzy, preand post-fMRI scan. Motor speed was measured with a box completion task and vigilance was measured with a numeral cancellation task (Lewis and Kupke, 1977).

Cognitive paradigm performed during $f M R I$ scanning. The $N$-back verbal working memory task varied by load (0-, 2-, 3-back) and trial type (nonlure, target, lure) (Fig. 1A). Subjects were presented with a series of single consonants that appeared on the screen every $2 \mathrm{~s}$, one after the other. Using two buttons, subjects responded to every letter to indicate whether it matched or did not match the letter seen $n$ previously. A target was defined as a letter that matched the letter seen $n$-previously; for example, in a 3-back condition, the second R in the sequence R-T-K-R-D is the target. Lure trials matched a recently seen but nontarget letter. For example, in a 3-back condition, the second D in the sequence D-T-K$\mathrm{R}-\mathrm{D}$ is a lure; it matches the letter seen four letters earlier but not three, as the condition specifies. All other nontarget trials were categorized as nonlures. In contrast to targets and nonlures, lures create high interference as they give a spurious sense of familiarity, and place the greatest demand on cognitive control mechanisms (Gray et al., 2003). Previous data demonstrate that lure trials are uniquely sensitive to differences in PFC-dependent processes within and between groups (Perlstein et al., 2001, 2004) and individual differences in fluid intelligence (Gray et al., 
2003). Lure interference effects are also more pronounced in older adults, although target performance is unimpaired (Schmiedek et al., 2009).

On 0-back blocks, subjects indicated whether or not a target letter (X) appeared. Zero-back blocks consisted of targets and nontargets; lure trials were not relevant in this condition. The task was set up as 16 blocks of trials (eight 0-back blocks, four 2-back blocks, and four 3-back blocks) ordered in a Latin squares sequence. Subjects were instructed on the condition before each block of trials. A block consisted of 20 trials plus five randomly placed null events to introduce temporal jitter (necessary for event-related fMRI analyses). A trial consisted of a stimulus presented for $1 \mathrm{~s}$ followed by a $1 \mathrm{~s}$ delay. A null event was a $2 \mathrm{~s}$ blank period (the same duration as a regular trial). Trial types included targets $(20 \%)$, lures $(15 \%)$, and nonlures (65\%). Each block of trials (50 s) was preceded by instructions ( $6 \mathrm{~s})$ and fixation (10 s) and followed by a brief period of rest (20 s).

Genetic analysis. DNA extraction and analysis was conducted according to standard methods on samples obtained from informed, consenting subjects. The Ernest Gallo Clinic and Research Center Genomics Core (Emeryville, CA) performed genotyping of the COMT Val ${ }^{158} / \mathrm{Met}$ (rs4680) polymorphism with PCR using TaqMan technology (Applied Biosystems). The COMT genotype frequencies of prescreened subjects were in Hardy-Weinberg equilibrium $\left(\chi^{2}=2.01\right.$, df $=1$, not significant). The genotype distribution was as follows: $\mathrm{val} / \mathrm{val}, 26$ subjects; val/ met, 33 subjects; met/met, 20 subjects).

COMT enzyme analysis. One $4-5 \mathrm{ml}$ blood sample was collected in a vacutainer EDTA tube (BD Diagnostic Systems) on each test day by a licensed phlebotomist to measure COMT enzyme levels. Genotyping the $\mathrm{Val}^{158}$ Met polymorphism provides a static determination of genotype. COMT function is determined by the extent to which the gene gets translated into an active protein-the COMT enzyme. Enzymatic activity is strongly related to COMT genotype but can vary even within the static designations of $\mathrm{val} / \mathrm{val}$, val/met, and $\mathrm{met} / \mathrm{met}$. Assaying blood COMT enzyme levels provides an additional, more direct measure of COMT activity than assaying genotype alone and reflects brain COMT levels (Chen et al., 2004). COMT enzyme activity was determined in whole peripheral blood collected on each scan day. The COMT enzyme activity assay is based on the organic solvent extraction method that separates the radioactive product; the methylated catechol; and the free radioactive coenzyme, ${ }^{3} \mathrm{H}$-S-adenosyl-methionine, as described previously (Apud et al., 2007). Determination of whole-blood COMT activity confirmed that met/met samples $(N=14)$ had greatly reduced COMT activity $(23,408 \pm 2465 \mathrm{cpm}$ mg protein, mean \pm SEM) compared with $\mathrm{val} / \mathrm{val}$ samples $(N=23)\left(51,474 \pm 3688 \mathrm{cpm} \mathrm{mg}\right.$ protein; $t_{(35)}=5.480$; $p<0.0001)$. Variance in the amount of active COMT protein is evident within genotypic designations and is likely due to epigenetic or epistatic interactions.

Estradiol assay/menstrual cycle phase monitoring. One $4-5 \mathrm{ml}$ blood sample was collected in a vacutainer SST tube (BD Diagnostic Systems) on each test day by a licensed phlebotomist to measure serum estradiol levels. Samples were stored on ice until centrifugation, after which serum was collected and stored at $-20^{\circ} \mathrm{C}$ until assayed. $17 \beta$-Estradiol concentrations were determined using a commercially available enzyme immunoassay (ELISA; Calbiotech). The assay sensitivity was $10 \mathrm{pg} / \mathrm{ml}$ and the intra-assay coefficient of variation was $2.26 \%$. All samples were run in a single assay and all instructions provided by the manufacturer were followed without modification. Menses/early follicular phase testing was first confirmed from cycle records: all low estradiol test sessions occurred within $3 \mathrm{~d}$ of the start of menses (duration of menses across subjects ranged from 3 to $7 \mathrm{~d}$ ). Determination of serum estradiol levels confirmed that subjects tested during the early follicular phase had reduced levels of circulating estradiol $(137.5 \pm 58.9 \mathrm{pg} / \mathrm{ml})$ compared with the latefollicular phase $(251 \pm 178 \mathrm{pg} / \mathrm{ml})$. Thirty-seven samples were assayed. Samples from seven test sessions (four low estradiol sessions and three high estradiol sessions) were not assayed due to subject constraints (difficulty acquiring an adequate blood sample) or the sample being unusable (problems extracting serum from whole blood). A record of those subjects' menstrual cycles confirmed that the four low sessions for which blood samples were not assayed occurred while the subject was in menses.

fMRI data acquisition and analysis. MRI data were acquired with a Siemens 3T Trio Magnetom scanner. Functional data were obtained using a $\mathrm{T}^{*}$ weighted echo-planar imaging sequence sensitive to blood oxygenation level-dependent (BOLD) contrast (repetition time, 2000 $\mathrm{ms}$; echo time, $28 \mathrm{~ms}$; field of view, $230 \mathrm{~mm}$; flip angle, $90^{\circ}$; voxel size, $2.0 \times 1.8 \times 3.0$ ). Each functional volume consisted of $293 \mathrm{~mm}$ oblique axial slices separated by a $0.45 \mathrm{~mm}$ interslice gap. A high-resolution T1 (MPRAGE) anatomical scan was also acquired. Following acquisition, MRI data were converted to Nifti format; processing included correction for slice-timing and motion artifacts, spatial normalization, and spatial smoothing with a three-dimensional Gaussian kernel $(8 \mathrm{~mm}$ full-width half-maximum). For spatial normalization, the individual subject's MPRAGE was coregistered to the mean functional image and normalized to the Montreal Neurological Institute (MNI) structural template. Normalization parameters were then written to the functional images. Time series were high-pass filtered ( $240 \mathrm{~s}$ cutoff). Statistical parametric maps of BOLD activation were calculated in SPM5 (http://www.fil.ion.ucl.ac.uk/ spm) using the general linear model approach (Worsley and Friston, 1995).

The main analysis focused on BOLD activity in load-sensitive regions within PFC. Statistical maps representing areas with linear increases of activity across memory load (3->2->0-back) were generated at the random effects level (family-wise error $p$ value $<0.00001$ ). The most significant load-sensitive PFC region (that which exceeded a threshold of $\mathrm{T}=8$ ) was within middle frontal gyrus (MFG). Thus, this region was the focus of our current analyses, consistent with previous COMT $N$-back findings (Egan et al., 2001; Mattay et al., 2003), which localize COMT genotype effects to dorsolateral PFC. To capture the true linear relationship between load conditions, the conditions were weighted in the contrast as follows: 0-back (-5/3), 2-back (1/3), 3-back (4/3). Bilateral PFC regions of interest (ROIs) were defined as $10 \mathrm{~mm}$ spheres around peak loci. The statistical model was then reapplied to the average BOLD signal within the ROI using MarsBar (www.mrc-cbu.cam.ac.uk/imaging/marsbar. $\mathrm{html}$ ). Mean beta weights from the ROIs defined from the load contrast were extracted and compared directly between genotype (val, met) and estradiol (low, high) groups and entered into an ANOVA to examine estradiol $\times$ genotype effects. Event-related analyses modeled only correct trials to avoid error-related confounds.

To obtain additional evidence for a relationship between DA and sustained PFC function, we entered COMT enzyme as a covariate of interest at the group level. Whole-brain maps revealed a specific, significant relationship between COMT enzymatic activity and left PFC activity, with no other association across the brain. To probe this finding further in an unbiased manner, we used an independently defined left and right PFC ROI from a meta-analysis of $N$-back activations observed across $24 \mathrm{fMRI}$ studies (Owen et al., 2005) and reapplied the statistical model (parametric load contrast) to the average signal across the ROI $[10 \mathrm{~mm}$ sphere around coordinates; left dorsolateral PFC (dlPFC): $-44,17,25$; right dlPFC: $40,31,34$; MNI space) for each subject's session. Data from the left and right PFC ROI were extracted and the mean signal values were submitted to Pearson's product-moment correlation.

\section{Results \\ Predictions}

We predicted that val/val subjects (low baseline DA) would show improved WM performance when estradiol levels were high versus low, whereas met/met subjects (high DA) would show the opposite pattern, with the best performance occurring when estradiol levels were low (Fig. $1 B$ ). These predictions were based on pharmacological COMT studies showing that val/val individuals improve on PFC-dependent tasks after administration of a DA agonist, whereas met/met individuals are impaired by the same drug (Egan et al., 2001). Considering the estrogen-cognition relationship within a model of PFC DA function allows us to address, with more precision, how estradiol impacts DAdependent neural processes. Demand for cognitive control dur- 
ing the WM task increases across trial type, from nonlures (lowest demand) to targets to lures (highest demand) (Gray et al., 2003) and across load (Braver et al., 1997). Given evidence that increased WM demands lead to increased synaptic DA release in PFC (Watanabe et al., 1997; Phillips et al., 2004; Aalto et al., 2005), a further prediction was that the most pronounced gene/ hormone effects would occur during lure trials or during highload blocks, when cognitive control demands are highest. At a neural level, we predicted that increased synaptic DA would be associated with greater neural efficiency (i.e., at an equal level of performance, a high DA state would increase the signal-to-noise ratio of cortical processing, decreasing the extent of PFC activity), in keeping with previous COMT and patient findings (Egan et al., 2001; Tunbridge et al., 2006).

\section{Subjective effects}

No differences were observed between genotypic groups (val, met) or estradiol status (low, high) on background demographic or neuropsychological measures (including age, education, Beck's Depression Inventory, Montreal Cognitive Assessment, Barratt Impulsiveness Scale, letter fluency, and North American Adult Reading Test), or measures of mood (visual analog scales for the items anxious, happy, sad, nauseous, drowsy, jittery, fatigued, and dizzy), affect (positive and negative affect scale), motor speed, and vigilance administered before and after MRI scanning on each test day.

\section{Behavioral results}

Parametric effects of $N$-back WM load (Fig. 2A) and trial type (Fig. $2 B$ ) were strongly reflected in performance. No group differences were observed for overall task performance (i.e., blocklevel performance). The overall mean accuracies (response times) across groups were as follows: $\mathrm{val} / \mathrm{val}, 75.9 \%$ (546.3 ms); met/met, $75.6 \%$ (560.8 ms); low estradiol, 76.1\% (561.9); high estradiol, $75.4 \%$ (547.4 ms). At the trial level, group differences in performance were not significant when examining estradiol status (low, high) and genotype (val, met) alone. Performance differences emerged when examining the impact of estradiol and genotype together, as predicted. On the high-interference/highcontrol lure trials during the 2-back condition, $\mathrm{val} / \mathrm{val}$ women performed poorly at the start of their cycle when estradiol was low and improved when estradiol levels were high. met/met women showed the opposite pattern, with strong performance under low estradiol conditions and impaired performance under high estradiol conditions (Fig. 2C). Comparing the low DA (val, low estradiol) and optimal DA (met, low estradiol) groups shows a significant difference in 2-back lure accuracy. In the 3-back condition, lure performance was poor across all subjects (mean accuracy: 2-back lure, $84 \%$; 3-back lure, 67\%) and the effects were not significant as a function of genotype. Of note, the 3-back lure data appear to fit the inverted U-shaped theoretical model (Fig. $1 B$ ) if the predicted task-induced shift in DA (Watanabe et al., 1997; Phillips et al., 2004; Aalto et al., 2005) is weighted more strongly (i.e., for the condition that places the greatest demand on cognitive control, 3-back lures, val and met groups are shifted farther along the DA axis).

We also examined lure accuracy within low and high estradiol groups as a function of COMT enzyme level (which provides a measure of the biologically active protein above and beyond static genotype) (Fig. 2D). For high estradiol subjects, COMT enzyme activity was positively correlated with lure accuracy; for low estradiol subjects, enzyme levels were negatively correlated with lure accuracy (Fig. 2E). This behavioral finding is consistent with
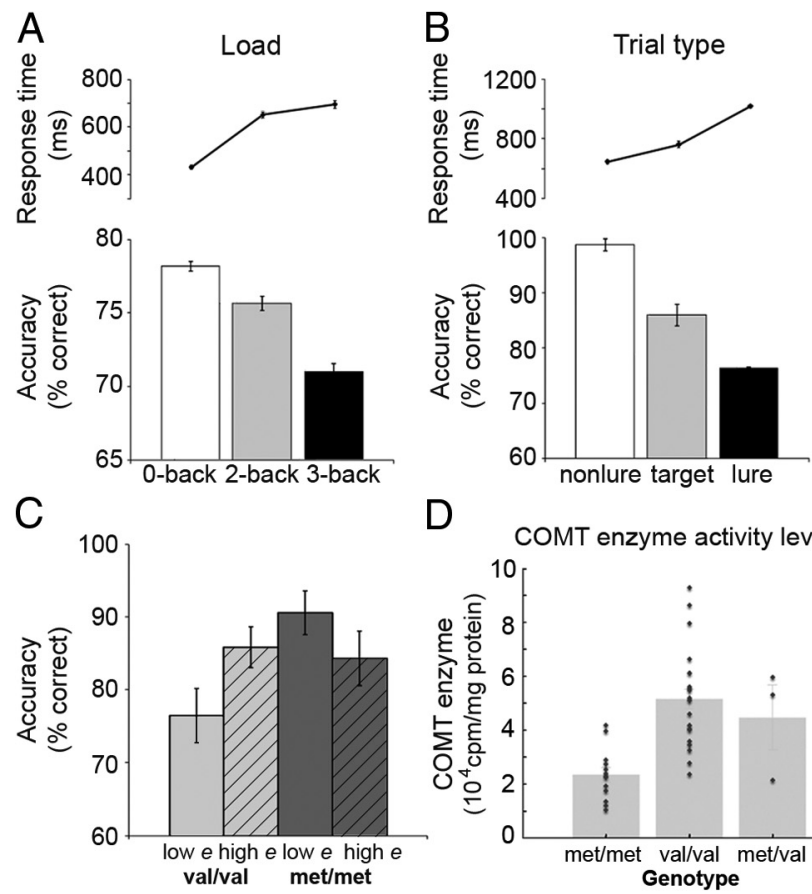

D
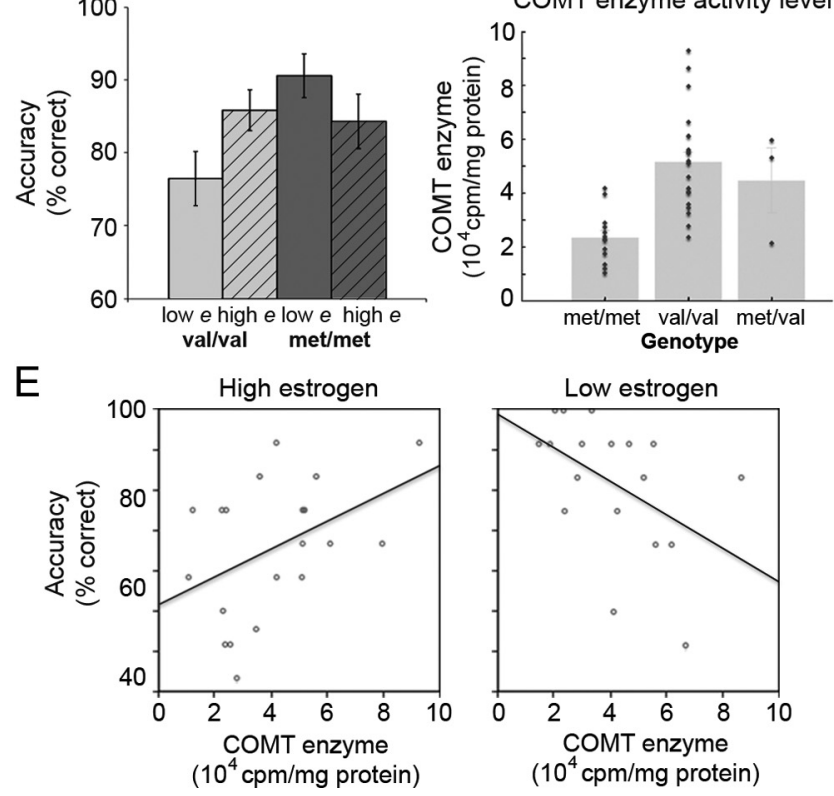

COMT enzyme activity leve

Figure 2. $\quad A$, Repeated-measures one-way ANOVA shows a significant difference in load $\operatorname{accuracy}\left(F_{(2,86)}=93.98 ; p<0.0005\right.$; effect size, $\left.\eta^{2}=0.69\right)$ and response time $\left(F_{(1,43)}=401.63\right.$, $\left.p<0.0005, \eta^{2}=0.90\right)$. Post hoc Bonferroni-corrected comparisons showed that all three means for both accuracy and response time were significantly different; subjects performed faster and more accurately on 0-back (accuracy, 78\%; response time, $436 \mathrm{~ms}$ ) compared with 2-back $(75.6 \%, 653 \mathrm{~ms})$ compared with 3-back $(71 \%, 696 \mathrm{~ms})$ trials. Error bars represent SEM. $N=44$. $B$, Similar analyses revealed significant differences in trial type accuracy $\left(F_{(1,43)}=81.14 ; p<0.0005\right.$; effect size, $\left.\eta^{2}=0.65\right)$ and response time $\left(F_{(1,43)}=\right.$ $\left.362.78 ; p<0.0005 ; \eta^{2}=0.89\right)$ : nonlure $(99 \%, 644 \mathrm{~ms})$, target $(86 \%, 759 \mathrm{~ms})$, lure (76\%, $1019 \mathrm{~ms})$. C, Two-back lure trial performance as a function of genotype $[N=16$ (met) 24 (val) $]$ and estradiol status $[N=21$ (high) 20, (low)]. met/met + low estradiol (e) subjects $(91 \% \pm 2.9 \%$ correct; mean \pm SEM) performed significantly better than val/val + low estradiol subjects (77\% $\pm 3.7 \% ; p=0.041$, two-tailed $t$ test). $\boldsymbol{D}$, COMT enzyme activity level by genotype, depicting variance within met/met and val/val genotypes (overlaid on mean values; pale gray bars). Blood samples from three heterozygous val/met samples were analyzed and are included for display only. $\boldsymbol{E}$, For low estradiol subjects (right), COMT enzyme correlates negatively with 2-back lure accuracy $(r=-0.482 ; p=0.043)$. For high estradiol subjects (left), COMT enzyme correlates positively with 3-back lure accuracy (approached significance $r=$ $0.433 ; p=0.056)$.

an inverted U-shaped function at the individual subject level. That is, just before ovulation, when estradiol levels are elevated, the best performers were women with high COMT activity (indicative of low baseline PFC DA). Conversely, toward the beginning of the cycle, when estradiol levels are reduced, the best performers were women with low COMT activity (high baseline PFC DA), in keeping with DA's inverted U-shaped action (Egan et al., 2001; Mattay et al., 2003). 

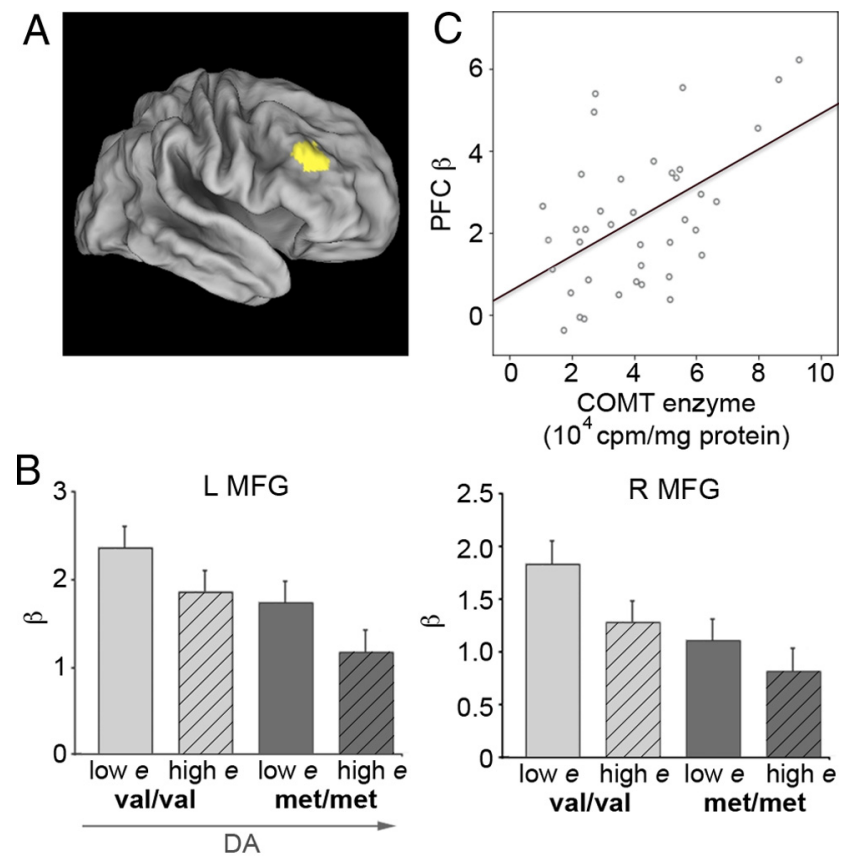

Figure 3. Across blocks, sustained PFC activity decreases as PFC DA increases across groups. $\boldsymbol{A}$, Load-sensitive suprathreshold PFC cluster (right hemisphere shown at family-wise error $p$ value $<$ $0.0001 ; T=7.5$ ). Left (L) and right ( $R$ ) MFG were identified as ROIs (10 contiguous voxels; MNI coordinates of peak locus, left: $-45,32,30$; right: 44,32, 32; defined at family-wise error $p$ value $<$ $0.00001) . N=44 . B$, Parameter estimates in bilateral MFG shown together as a function of genotype and estradiol (e) status (lowest vs highestDA groups: val + low estradiol vs met + high estradiol; left MFG, $p=0.021$; right MFG, $p=0.019$ ). C, Mean signal in left MFG versus COMT enzymatic activity $(N=37)$. As COMT activity increased (indicating a probable concomitant decrease in PFC DA), PFC activity became more exaggerated.

\section{Functional MRI results}

To investigate the neural effects underlying hormone-genotype interactions, we examined BOLD activity in load-sensitive PFC regions, which reflects areas involved in WM maintenance processes. Despite the observation that behavioral performance across loads did not differ between groups, by examining PFC activity sustained across task blocks, we found that bilateral MFG activity markedly declined as inferred PFC DA increased. Higher DA (high estradiol, met genotype, or both) was associated with lower sustained PFC activation, whereas lower DA (low estradiol, val genotype, or both) was associated with greater sustained PFC activation (Fig. 3B). Parameter estimates, or mean beta values, in right MFG (Fig. $3 A$ ) differed as a function of genotype (val, met) $(p=0.038)$ and estradiol status (low, high) $(p=0.011)$. Similar results were observed in left MFG.

Furthermore, average signal magnitude in left MFG correlated with COMT activity $(r=0.513, p<0.001$; right MFG did not reach significance) (Fig. $3 C$ ), suggesting that, as the amount of DA available in PFC declines (that is, as COMT activity increases), PFC activity is enhanced.

We examined activity during high-interference/high-control lure trials and lower-interference target trials within the WM load-sensitive PFC ROIs. Target-related activity showed the same pattern of decreased bilateral MFG activity with increased PFC DA as observed at the sustained-activity block level (Fig. 4A). Lure trials showed a different pattern (Fig. $4 B$ ), where the magnitude of MFG activation mirrored the inverted U-shaped performance curve (i.e., there was a selective increase in activity for higher, more optimal DA groups: val/val plus low estradiol compared with met/met plus low estradiol; $p=0.028)$. Moreover, the greater a subject's MFG response to lure trials, the better their
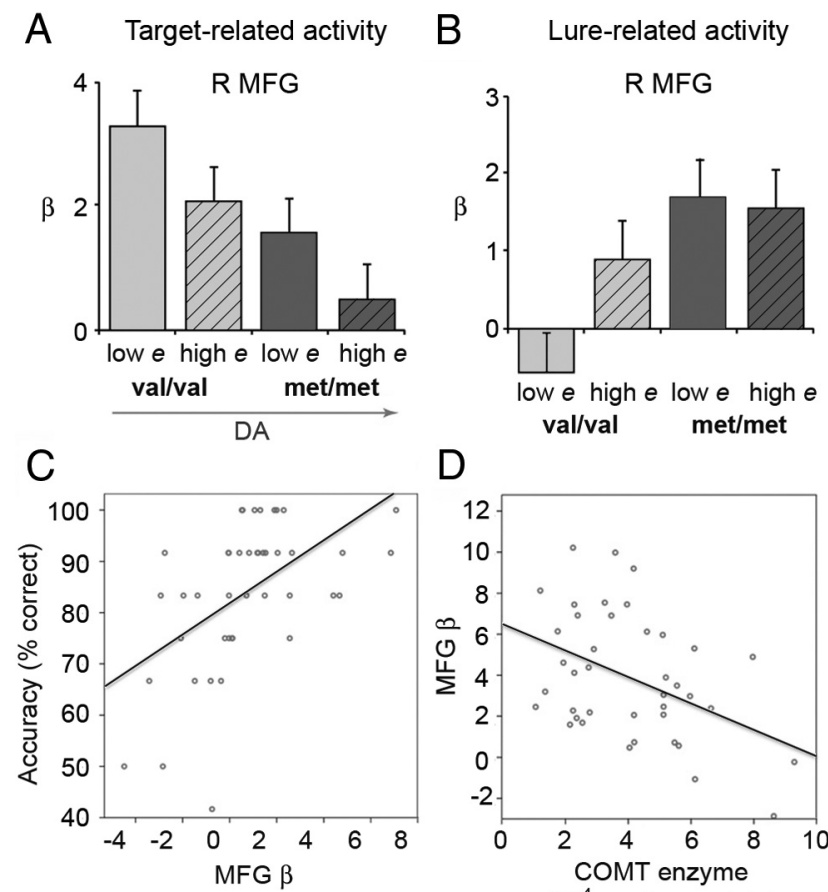

D

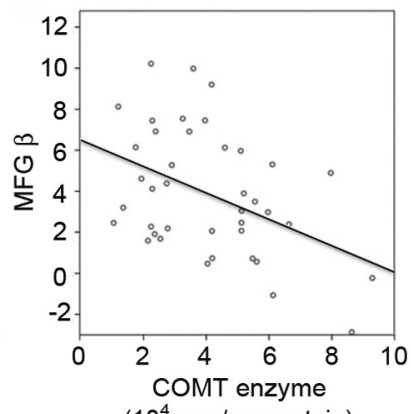

$\left(10^{4} \mathrm{cpm} / \mathrm{mg}\right.$ protein)

Figure 4. $\quad \boldsymbol{A}, \boldsymbol{B}$, Target-related $(\boldsymbol{A})$ and lure-related $(\boldsymbol{B})$ neural activity as a function of inferred PFC dopamine status (event-related activity on correct trials within 2-and 3-back WM blocks; note that 0 -backblocks do not, by necessity, contain lure trials). C, Two-back lure performance versus the magnitude of correct lure-trial activity in right (R) MFG (mean signal within the ROI). D, COMT enzyme versus the magnitude of correct lure-trial activity in right MFG (mean signal).e, Estradiol.

lure performance (2-back lures: $r=0.532, p<0.005$; total lures: $r=0.392, p=0.009$ ) (Fig. $4 C$ ).

To further probe the connection between PFC DA, MFG activity, and lure performance at the single subject level, we compared COMT enzyme with lure activity within these PFC ROIs. In right MFG, activity during lure trials was negatively correlated with COMT enzyme $(r=0.425, p=0.006$; the association did not meet significance within the left MFG ROI) (Fig. $4 D$ ). In the right MFG, the relationship was significant for both 2-back ( $r=$ $0.334, p=0.035)$ and 3-back $(r=0.4, p=0.01)$ lure trials.

In summary, the fMRI findings suggest that cortical DA is associated with a broadly efficient pattern of sustained activity (that which occurs across WM blocks), and a selective, event-related enhancement of activity during episodes of high interference (e.g., lures), when the demand for cognitive control is greatest. Furthermore, the extent to which an individual enhances PFC activation during the demanding lure trials is predictive of their performance.

\section{Discussion}

In humans, there is a strong effort to understand the effects of estrogen on cognition, but the data have been inconsistent. This study suggests that taking baseline DA into account is crucial for detecting the direction of estrogen's effect on WM. Specifically, the results establish that estradiol can be beneficial or detrimental to WM and, critically, the direction of the effect depends on COMT genotype and, on a finer scale, COMT enzymatic activity (proxies of baseline PFC DA). At the behavioral level, the effects of estradiol and COMT genotype emerged on trials that required a high demand of cognitive control. Neurally, hormonal and genotypic differences were apparent even in the absence of significant behavioral differences. Suboptimal DA subjects showed greater task-related PFC activity across blocks but reduced PFC 
activity on the high interference lure trials. Optimal DA subjects showed the opposite pattern. Conceptually similar results with the same cognitive task were observed in a study of the neural mechanisms of general fluid intelligence (gF) (Gray et al., 2003), in which low gF subjects (like our suboptimal DA subjects) showed enhanced sustained PFC activity during a WM task but paradoxically reduced activity during high-control interference trials. High gF individuals showed the opposite pattern (like our optimal DA subjects).

Our findings are in keeping with work from Egan et al. (2001), who demonstrated that Met allele load predicts the efficiency of PFC physiology. Although all genotypic groups in their sample ( $\mathrm{val} / \mathrm{val}$, $\mathrm{val} / \mathrm{met}$, met/met) showed similar levels of performance on a 2-back WM task, task-related PFC BOLD activity was greatest for $\mathrm{val} / \mathrm{val}$ subjects, reduced for $\mathrm{val} / \mathrm{met}$, and lowest for met/met subjects. In a related pharmacogenomic study, Mattay et al. (2003) found that amphetamine administration improved WM performance and enhanced cortical efficiency for $\mathrm{val} / \mathrm{val}$ subjects. met/met subjects, however, became cortically inefficient on drug, presumably because near-optimal basal DA levels were heightened beyond the optimal range. Similarly, Mehta et al. (2000) showed that the indirect catecholamine agonist methylphenidate (Ritalin) improves WM performance and reduces task-related regional cerebral blood flow in the PFC. These beneficial drug effects were most pronounced for subjects with lower baseline WM capacities (a likely behavioral index of DA synthesis capacity) (Cools et al., 2008). Similar results were found using the D2 receptor agonist bromocriptine (Kimberg et al., 1997; Gibbs and D'Esposito, 2005b; Cools et al., 2007). Together, these findings contribute to a body of evidence demonstrating that optimal DA levels are associated with greater cortical efficiency (i.e., at a given level of performance, DA optimizes the signal-to-noise ratio of cortical processing, decreasing the extent of task-related PFC activity as measured by BOLD) (Durstewitz and Seamans, 2002; Winterer and Weinberger, 2004; Vijayraghavan et al., 2007). The relationship between DA, WM, and cortical efficiency has been demonstrated in humans using pharmacological manipulation of DA; we observe a strikingly similar relationship when considering the natural hormonal fluctuations that occur over the coarse of a woman's menstrual cycle. Further, we found that estradiol's DA agonist-like effect on behavioral and neural processes depends on COMT Val ${ }^{158}$ Met genotype and, at an individual level, COMT enzymatic activity, which suggests a dependence on baseline PFC DA.

The importance of ovarian hormones to the neurophysiology underlying prefrontal function was established by Berman and colleagues (1997). The authors examined the influence of gonadal hormones on regional cerebral blood flow ( $\mathrm{rCBF}$ ) by suppressing endogenous ovarian activity and reintroducing exogenous estradiol and progesterone. Task-evoked prefrontal rCBF patterns were dramatically reduced under hormonesuppression conditions and normalized after hormone replacement. This paradigm provides excellent control for the manipulation of gonadal hormones. Menstrual cycle studies depend on the endogenous fluctuation of sex hormones, and the timing of hormone fluctuation varies between women and between cycles. In the present study, women were tracked for $\geq 4$ months to confirm the consistency of their cycle and to infer hormone status at the time of testing (supplemented by direct estradiol measurement from serum samples), but this paradigm is limited in its ability to tease apart the concerted action of multiple sex hormones.

Norepinephrine (NE) also plays a key role in WM mechanisms (Li and Mei, 1994; Li et al., 1999; Ramos et al., 2005) and our results do not rule out the possibility that NE signaling, and modulation thereof, is part of estradiol's underlying mechanism for modulating PFC function. However, in this study, the effects of estradiol were highly dependent on COMT. COMT inactivates catecholamines, including DA and NE, via $o$-methylation (Männistö and Kaakkola, 1999). Evidence suggests that COMT's role in metabolizing NE in PFC is minor compared with its role in DA metabolism. Administration of tolcapone, a brain-penetrant COMT inhibitor, enhances extracellular DA in rat medial PFC with little to no change in NE (measured following stimulation of the catecholamine system) (Tunbridge et al., 2004). Furthermore, Huotari et al. (2002) observed that, following L-DOPA administration, COMT knock-out mice show increased accumulation of cortical DA compared with wild-type animals; cortical NE levels did not differ significantly across groups. Together, these data suggest that COMT predominantly impacts PFC DA, with minimal influence over NE signaling.

$\mathrm{GABA}$, the principle inhibitory neurotransmitter, has also been shown to fluctuate over the menstrual cycle (Epperson et al., 2002; for review, see Cosgrove et al., 2007) and mounting evidence demonstrates that estradiol modulates glutamatergic activity (McCarthy et al., 2002). Thus, estradiol's influence on cognition cannot be fully understood without taking other neurochemical systems into account. Moreover, estradiol does not exert its effect in isolation; other gonadal hormones such as progesterone likely modulate PFC function (Berman et al., 1997). In vitro and in vivo experimental paradigms in animals and carefully designed studies in humans are critical for clarifying the complex relationship between endogenous fluctuations in neuroactive gonadal hormones, neurochemical signaling, and cognition (Aubele and Kritzer, 2011).

The results of our study are in keeping with work in rodents and nonhuman primates showing that estradiol enhances DA activity, but a definitive study showing an estradiol-DA link in humans is lacking. Further studies are needed to directly assess estradiol's effect on DA neurotransmission in humans. The only method currently available to assess DA release in vivo in humans combines pharmacological stimulation of the DA system with positron emission tomography, in conjunction with a DA-receptor radioligand such as $\left[{ }^{11} \mathrm{C}\right]$-raclopride (Laruelle, 2000; Cropley et al., 2006).

Although no study has compared estradiol levels (Bixo et al., 1995 ) or the pattern of estrogen receptor (ER) expression across specific subregions of PFC in humans, quantitative morphometric studies in nonhuman primates demonstrate estradiol's influence on synaptic organization within dorsolateral PFC (Kritzer and Kohama, 1998), a region that expresses ER $\alpha$ (Perlman et al., 2005; Montague et al., 2008; Wang et al., 2010). Specifically, when ovariectomized monkeys were treated with estradiol (using a cyclical regimen that mirrors the preovulatory spike in estradiol), striking increases in dendritic spine density were observed in dlPFC layer III pyramidal neurons (Hao et al., 2006). These physiological changes were accompanied by improved performance on a delayed-response working-memory task (Rapp et al., 2003). Importantly, the estradiol-dependent effects we observed in humans using fMRI BOLD are also in dlPFC. Thus, the relationship between estradiol, DA, and working memory is underscored by the overlapping neural proximity with which these changes are observed.

Another important clue into estradiol's site of action comes from a series of immunocytochemistry experiments showing ER $\beta$ expression in midbrain dopaminergic neurons that project to PFC and constitute the mesocortical DA pathway (Creutz and Kritzer, 2002). Until recently it was thought that $\sim 30 \%$ of cells within the rat me- 
socortical pathway were dopaminergic ( $\mathrm{TH}$-immunoreactive) (Swanson, 1982). However, the data came solely from males and a recent study discovered that females have a significantly greater proportion of dopaminergic cells, $\sim 50 \%$, within this pathway (Kritzer and Creutz, 2008). Sex differences in mesocortical organization may carry functional consequences for cognitive processes that rely on PFC DA signaling.

Collectively, these data carry direct ramifications for women's health. The response to DA medications (e.g., Ritalin for attention deficit disorder and L-DOPA for Parkinson's disease) may differ between men and women, and within women in different endocrine states (Lukas et al., 1996; Justice and deWit, 1999, 2000; Evans et al., 2002). A substantial body of evidence from the animal and human literature documents sex differences in neural and behavioral responses to dopaminergic drugs, including pharmacological treatments and drugs of abuse (Goldstein et al., 2002; Lynch et al., 2002; Carroll et al., 2004; Becker and $\mathrm{Hu}, 2008$ ). The present findings suggest that although estrogen, considered in isolation, may have unpredictable effects on cognition, its influence is clarified when considered within a larger neuromodulatory framework. A man and woman's milieu differ; until we understand how, we cannot fully understand neural processes as they unfold in the healthy state, much less in the diseased state.

\section{References}

Aalto S, Brück A, Laine M, Någren K, Rinne JO (2005) Frontal and temporal dopamine release during working memory and attention tasks in healthy humans: a positron emission tomography study using the high-affinity dopamine $\mathrm{D}_{2}$ receptor ligand $\left[{ }^{11} \mathrm{C}\right] \mathrm{FLB} 457$. J Neurosci 25:2471-2477.

Apud JA, Mattay V, Chen J, Kolachana BS, Callicott JH, Rasetti R, Alce G, Iudicello JE, Akbar N, Egan MF, Goldberg TE, Weinberger DR (2007) Tolcapone improves cognition and cortical information processing in normal human subjects. Neuropsychopharmacology 32:1011-1020.

Aubele T, Kritzer MF (2011) Gonadectomy and hormone replacement affects in vivo basal extracellular dopamine levels in the prefrontal cortex but not motor cortex of adult male rats. Cereb Cortex 21:222-232.

Barrett LF, Tugade MM, Engle RW (2004) Individual differences in working memory capacity and dual-process theories of the mind. Psychol Bull 130:553-573.

Beck AT, Ward CH, Mendelson M, Mock J, Erbaugh J (1961) An inventory for measuring depression. Arch Gen Psychiatry 4:561-571.

Becker JB (1990) Estrogen rapidly potentiates amphetamine-induced striatal dopamine release and rotational behavior during microdialysis. Neurosci Lett 118:169-171.

Becker JB (2000) Oestrogen effects on dopaminergic function in striatum. In Neuronal and cognitive effects of oestrogens (Chadwick DJ, Goode JA, eds), pp. 134-145. West Sussex, England: Wiley.

Becker JB, Hu M (2008) Sex differences in drug abuse. Front Neuroendocrinol 29:36-47.

Benton AL (1968) Differential behavioral effects in frontal lobe disease. Neuropsychologia 6:53-60.

Berman KF, Schmidt PJ, Rubinow DR, Danaceau MA, Van Horn JD, Esposito G, Ostrem JL, Weinberger DR (1997) Modulation of cognition-specific cortical activity by gonadal steroids: a positron-emission tomography study in women. Proc Natl Acad Sci U S A 94:8836-8841.

Bixo M, Bäckström T, Winblad B, Andersson A (1995) Estradiol and testosterone in specific regions of the human female brain in different endocrine states. J Steroid Biochem Mol Biol 55:297-303.

Braver T, Cohen JD, Nystrom LE, Jonides J, Smith EE, Noll DC (1997) A parametric study of prefrontal cortex involvement in human working memory. Neuroimage 5:49-62.

Brozoski TJ, Brown RM, Rosvold HE, Goldman PS (1979) Cognitive deficit caused by regional depletion of dopamine in prefrontal cortex of rhesus monkey. Science 205:929-932.

Cai JX, Arnsten AF (1997) Dose-dependent effects of the dopamine D1 receptor agonists A77636 or SKF81297 on spatial working memory in aged monkeys. J Pharmacol Exp Ther 283:183-189.
Carroll ME, Lynch WJ, Roth ME, Morgan AD, Cosgrove KP (2004) Sex and estrogen influence drug abuse. Trends Pharmacol Sci 25:273-279.

Chen J, Lipska BK, Halim N, Ma QD, Matsumoto M, Melhem S, Kolachana BS, Hyde TM, Herman MM, Apud J, Egan MF, Kleinman JE, Weinberger DR (2004) Functional analysis of genetic variation in catechol-Omethyltransferase (COMT): effects on mRNA, protein, and enzyme activity in postmortem human brain. Am J Hum Genet 75:807-821.

Cools R, Sheridan M, Jacobs E, D’Esposito M (2007) Impulsive personality predicts dopamine-dependent changes in frontostriatal activity during component processes of working memory. J Neurosci 27:5506-5514

Cools R, Gibbs SE, Miyakawa A, Jagust W, D’Esposito M (2008) Working memory capacity predicts dopamine synthesis capacity in the human striatum. J Neurosci 28:1208-1212.

Cosgrove KP, Mazure CM, Staley JK (2007) Evolving knowledge of sex differences in brain structure, function, and chemistry. Biol Psychiatry 62:847-855.

Creutz LM, Kritzer MF (2002) Estrogen receptor- $\beta$ immunoreactivity in the midbrain of adult rats: regional, subregional and cellular localization in the A10, A9, and A8 dopamine cell groups. J Comp Neurol 446:288-300.

Cropley VL, Fujita M, Innis RB, Nathan PJ (2006) Molecular imaging of the dopaminergic system and its association with human cognitive function. Biol Psychiatry 59:898-907.

Daneman M, Carpenter PA (1980) Individual differences in working memory and reading. J Verb Learn Verb Behav 19:450-466.

Duff SJ, Hampson E (2000) A beneficial effect of estrogen on working memory in postmenopausal women taking hormone replacement therapy. Horm Behav 38:262-276.

Durstewitz D, Seamans JK (2002) The computational role of dopamine D1 receptors in working memory. Neural Netw 15:561-572.

Egan MF, Goldberg TE, Kolachana BS, Callicott JH, Mazzanti CM, Straub RE, Goldman D, Weinberger DR (2001) Effect of COMT Val108/158 Met genotype on frontal lobe function and risk for schizophrenia. Proc Natl Acad Sci U S A 98:6917-6922.

Epperson CN, Haga K, Mason GF, Sellers E, Gueorguieva R, Zhang W, Weiss E, Rothman DL, Krystal JH (2002) Cortical $\gamma$-aminobutyric acid levels across the menstrual cycle in healthy women and those with premenstrual dysphoric disorder. Arch Gen Psychiatry 59:851-858.

Evans SM, Haney M, Foltin RW (2002) The effects of smoked cocaine during the follicular and luteal phases of the menstrual cycle in women. Psychopharmacology 159:397-406.

Gasbarri A, Pompili A, d'Onofrio A, Cifariello A, Tavares MC, Tomaz C (2008) Working memory for emotional facial expressions: role of the estrogen in young women. Psychoneuroendocrinology 33:964-972.

Gibbs SE, D’Esposito M (2005a) Individual capacity differences predict working memory performance and prefrontal activity following dopamine receptor stimulation. Cogn Affect Behav Neurosci 5:212-221.

Gibbs SE, D'Esposito M (2005b) A functional MRI study of the effects of bromocriptine, a dopamine receptor agonist, on component processes of working memory. Psychopharmacology 180:644-653.

Goldstein JM, Cohen LS, Horton NJ, Lee H, Andersen S, Tohen M, Crawford A, Tollefson G (2002) Sex differences in clinical response to olanzapine compared with haloperidol. Psychiatry Res 110:27-37.

Granon S, Passetti F, Thomas KL, Dalley JW, Everitt BJ, Robbins TW (2000) Enhanced and impaired attentional performance after infusion of D1 dopaminergic receptor agents into rat prefrontal cortex. J Neurosci 20:1208-1215.

Gray JR, Chabris CF, Braver TS (2003) Neural mechanisms of general fluid intelligence. Nat Neurosci 6:316-322.

Hao J, Rapp PR, Leffler AE, Leffler SR, Janssen WG, Lou W, McKay H, Roberts JA, Wearne SL, Hof PR, Morrison JH (2006) Estrogen alters spine number and morphology in prefrontal cortex of aged female rhesus monkeys. J Neurosci 26:2571-2578.

Huotari M, Gogos JA, Karayiorgou M, Koponen O, Forsberg M, Raasmaja A, Hyttinen J, Männistö PT (2002) Brain catecholamine metabolism in catechol-o-methyltransferase (COMT)-deficient mice. Eur J Neurosci $15: 246-256$.

Justice AJ, de Wit H (1999) Acute effects of d-amphetamine during the follicular and luteal phases of the menstrual cycle in women. Psychopharmacology 145:67-75.

Justice AJ, de Wit H (2000) Acute effects of d-amphetamine during the early 
and late follicular phases of the menstrual cycle in women. Pharmacol Biochem Behav 66:509-515.

Kimberg DY, D'Esposito M, Farah MJ (1997) Effects of bromocriptine on human subjects depend on working memory capacity. Neuroreport 8:3581-3585.

Kritzer MF, Creutz LM (2008) Region and sex differences in constituent dopamine neurons and immunoreactivity for intracellular estrogen and androgen receptors in mesocortical projects in rats. J Neurosci 28:9525-9535.

Kritzer MF, Kohama SG (1998) Ovarian hormones influence the morphology distribution and density of tyrosine hydroxylase immunoreactive axons in the dorsolateral prefrontal cortex of adult Rhesus monkeys. J Comp Neurol 395:1-17.

Laruelle M (2000) Imaging synaptic neurotransmission with in vivo binding competition techniques: a critical review. J Cereb Blood Flow Metab 20:423-451.

Lewis R, Kupke T (1977) The Lafayette Clinic repeatable neuropsychological test battery: its development and research applications. Paper presented at the Southeastern Psychological Association, Hollywood, FL, May

Li BM, Mei ZT (1994) Delayed response deficit induced by local injection of the alpha-2 adrenergic antagonist yohimbine into the dorsolateral prefrontal cortex in young adult monkeys. Behav Neural Biol 62:134-139.

Li BM, Mao ZM, Wang M, Mei ZT (1999) Alpha-2 adrenergic modulation of prefrontal cortical neuronal activity related to spatial working memory in monkeys. Neuropsychopharmacology 21:601-610.

Lukas SE, Sholar M, Lundahl LH, Lamas X, Kouri E, Wines JD, Kragie L, Mendelson JH (1996) Sex differences in plasma cocaine levels and subjective effects after acute cocaine administration in human volunteers. Psychopharmacology (Berl) 125:346-354.

Lynch WJ, Roth ME, Carroll ME (2002) Biological basis of sex differences in drug abuse: preclinical and clinical studies. Psychopharmacology 164:121-137.

Männistö PT, Kaakkola S (1999) Catechol-O-methyltransferase (COMT): biochemistry, molecular biology, pharmacology and clinical efficacy of the new selective COMT inhibitors. Pharmacol Rev 51:593-628.

Mattay VS, Goldberg TE, Fera F, Hariri AR, Tessitore A, Egan MF, Kolachana B, Callicott JH, Weinberger DR (2003) Catechol O-methyltransferase val158-met genotype and individual variation in the brain response to amphetamine. Proc Natl Acad Sci U S A 100:6186-6191.

McCarthy MM, Auger AP, Perrot-Sinal TS (2002) Getting excited about GABA and sex differences in the brain. Trends Neurosci 25:307-312.

Mehta MA, Owen AM, Sahakian BJ, Mavaddat N, Pickard JD, Robbins TW (2000) Methylphenidate enhances working memory by modulating discrete frontal and parietal lobe regions in the human brain. J Neurosci 20:RC65.

Montague D, Weickert CS, Tomaskovic-Crook E, Rothmond DA, Kleinman JE, Rubinow DR (2008) Oestrogen receptor a localization in the prefrontal cortex of three mammalian species. J Neuroendocrinol 20:893-903.

Nasreddine ZS, Phillips NA, Bédirian V, Charbonneau S, Whitehead V, Collin I, Cummings JL, Chertkow H (2005) The Montreal cognitive assessment, MoCA: a brief screening tool for mild cognitive impairment. J Am Geriatr Soc 53:695-699.

Nelson HE (1982) National Adult Reading Test (NART) test manual. Windsor, UK: NFER-Nelson.

Owen AM, McMillan KM, Laird AR, Bullmore E (2005) N-Back working memory paradigm: a meta-analysis of normative functional neuroimaging studies. Hum Brain Mapp 25:46-59.

Pasqualini C, Olivier V, Guibert B, Frain O, Leviel V (1995) Acute stimulatory effect of estradiol on striatal dopamine synthesis. J Neurochem 65:1651-1657.

Patton JH, Stanford MS, Barratt ES (1995) Factor structure of the Barratt impulsiveness scale. J Clin Psychol 51:768-774.

Perlstein WM, Carter CS, Noll DC, Cohen JD (2001) Relation of prefrontal cortex dysfunction to working memory and symptoms in schizophrenia. Am J Psychiatry 158:1105-1113.

Perlstein WM, Cole MA, Demery JA, Seignourel PJ, Dixit NK, Larson MJ, Briggs RW (2004) Parametric manipulation of working memory load in traumatic brain injury: Behavioral and neural correlates. J Int Neuropsychol Soc 10:724-741.

Perlman WR, Matsumoto M, Beltaifa S, Hyde TM, Saunders RC, Webster MJ, Rubinow DR, Kleinman JE, Weickert CS (2005) Expression of estrogen- receptor alpha exon-deleted mRNA variants in the human and nonhuman primate frontal cortex. Neuroscience 134:81-95.

Phillips AG, Ahn S, Floresco SB (2004) Magnitude of dopamine release in medial prefrontal cortex predicts accuracy of memory on a delayed response task. J Neurosci 24:547-553.

Ramos BP, Colgan L, Nou E, Ovadia S, Wilson SR, Arnsten AF (2005) The beta- 1 adrenergic antagonist, betaxolol, improves working memory performance in rats and monkeys. Biol Psychiatry 58:894-900.

Rapp PR, Morrison JH, Roberts JA (2003) Cyclic estrogen replacement improves cognitive function in aged ovariectomized rhesus monkeys. J Neurosci 23:5708-5714.

Rosenberg L, Park S (2002) Verbal and spatial functions across the menstrual cycle in healthy young women. Psychoneuroendocrinology 27:835-841.

Salthouse TA, Babcock RL (1991) Decomposing adult age differences in working memory. Develop Psych 27:763-776.

Sawaguchi T, Goldman-Rakic PS (1991) D1 dopamine receptors in prefrontal cortex: involvement in working memory. Science 251:947-950.

Schmiedek F, Li SC, Lindenberger U (2009) Interference and facilitation in spatial working memory: age-associated differences in lure effects in the n-back paradigm. Psychol Aging 24:203-210.

Shansky RM, Glavis-Bloom C, Lerman D, McRae P, Benson C, Miller K, Cosand L, Horvath TL, Arnsten AF (2004) Estrogen mediates sex differences in stressinduced prefrontal cortex dysfunction. Mol Psychiatry 9:531-538.

Sherwin BB (2005) Estrogen and memory in women: how can we reconcile the findings? Horm Behav 47:371-375.

Stroop J (1935) Studies of interference in serial verbal reactions. J Exp Psychol 18:643-661.

Swanson LW (1982) The projections of the ventral tegmental area and adjacent regions: a combined fluorescent retrograde tracer and immunofluorescence study in the rat. Brain Res Bull 9:321-353.

Thompson TL, Moss RL (1994) Estrogen regulation of dopamine release in the nucleus accumbens: Genomic-and nongenomic-mediated effects. J Neurochem 62:1750-1756.

Tunbridge EM, Bannerman DM, Sharp T, Harrison PJ (2004) Catechol-omethyltransferase inhibition improves set-shifting performance and elevates stimulated dopamine release in the rat prefrontal cortex. J Neurosci 24:5331-5335.

Tunbridge EM, Harrison PJ, Weinberger DR (2006) Catechol-Omethyltransferase cognition and psychosis: Val158Met and beyond. Biol Psychiatry 60:141-151.

Unsworth N, Heitz RP, Schrock JC, Engle RW (2005) An automated version of the operation span task. Behav Res Methods 37:498-505.

Vijayraghavan S, Wang M, Birnbaum SG, Williams GV, Arnsten AF (2007) Inverted- $\mathrm{U}$ dopamine $\mathrm{D} 1$ receptor actions on prefrontal neurons engaged in working memory. Nat Neurosci 10:376-384.

Wang AC, Hara Y, Janssen WG, Rapp PR, Morrison JH (2010) Synaptic estrogen receptor-a levels in prefrontal cortex in female rhesus monkeys and their correlation with cognitive performance. J Neurosci 30:1277012776.

Watanabe M, Kodama T, Hikosaka K (1997) Increase of extracellular dopamine in primate prefrontal cortex during a working memory task. J Neurophysiol 78:2795-2798.

Watson D, Clark LA, Tellegen A (1988) Development and validation of brief measures positive and negative affect: the PANAS scales. J Pers Soc Psychol 54:1063-1070.

Williams GV, Goldman-Rakic PS (1995) Modulation of memory fields by dopamine D1 receptors in prefrontal cortex. Nature 376:572-575.

Williams-Gray CH, Hampshire A, Robbins TW, Owen AM, Barker RA (2007) Catechol $O$-methyltransferase val158met genotype influences frontoparietal activity during planning in patients with Parkinson's disease. J Neurosci 27:4832-4838.

Winterer G, Weinberger DR (2004) Genes, dopamine and cortical signalto-noise ratio in schizophrenia. Trends Neurosci 27:683-690.

Worsley KJ, Friston KJ (1995) Empirical analyses of BOLD fMRI statistics. Neuroimage 2:173-181.

Xiao L, Becker JB (1994) Quantitative microdialysis determination of extracellular striatal dopamine concentration in male and female rats: effects of estrous cycle and gonadectomy. Neurosci Lett 180:155-158.

Zahrt J, Taylor JR, Mathew RG, Arnsten AF (1997) Supranormal stimulation of D1 dopamine receptors in the rodent prefrontal cortex impairs spatial working memory performance. J Neurosci 17:8528-8535. 